\title{
Aspectos experimentales de la determinacion de curvas esfuerzo- deformacion a alta temperatura y en atmosfera controlada: Refractarios $\mathrm{Al}_{2} \mathrm{O}_{3}-\mathrm{MgO}-\mathrm{C}$
}

\author{
V. MUÑOZ; G.A. ROHR; A.G. TOMBA MARTINEZ; A.L. CAVALIERI \\ Instituto de Investigaciones en Ciencia y Tecnología de Materiales (INTEMA) \\ CONICET-Facultad de Ingeniería/Universidad Nacional de Mar del Plata \\ Av. J.B. Justo 4302 (7600) Mar del Plata, Argentina.
}

\begin{abstract}
Se presenta una metodología diseñada e implementada en el Laboratorio de Materiales Estructurales de la División Cerámicos del INTEMA para la evaluación del comportamiento mecánico de materiales refractarios a altas temperaturas y en atmósfera controlada. La metodología comprende la determinación de la deformación por extensometría de contacto en ensayos en compresión con el fin de obtener curvas esfuerzo-deformación y el uso de un sistema de control de atmósfera por desplazamiento a través del pasaje de una corriente gaseosa. La determinación de curvas esfuerzo-deformación de ladrillos refractarios comerciales $\mathrm{Al}_{2} \mathrm{O}_{3}-\mathrm{MgO}-\mathrm{C}$ utilizados en cucharas de acería a temperatura ambiente y a $1260{ }^{\circ} \mathrm{C}$ en diferentes atmósferas se presenta como ejemplo de aplicación de esta metodología.
\end{abstract}

Palabras clave: curvas esfuerzo-deformación, alta temperatura, atmósfera controlada, refractarios óxido-C

Experimental aspects of stress-strain curves determination at high temperature and controlled atmosphere: $\mathrm{Al}_{2} \mathrm{O}_{3}-\mathrm{MgO}-\mathrm{C}$ refractories

A methodology for the mechanical evaluation of refractory materials at high temperatures and controlled atmosphere, designed and implemented in the Structural Materials Laboratory of Ceramics Division of INTEMA, is described. The methodology includes the measurement of the specimen deformation by contact extensometry in compression tests to obtain stress-strain curves and the use of a gaseous flow as a system to control atmosphere. The determination of stress-strain curves of $\mathrm{Al}_{2} \mathrm{O}_{3}-\mathrm{MgO}-\mathrm{C}$ commercial refractories used in steelmaking ladles at room temperature and $1260^{\circ} \mathrm{C}$ in different atmospheres is presented as an example of application of this methodology.

Keywords: stress-strain curves, high temperature, controlled atmosphere, oxide-C refractories

\section{INTRODUCCION}

Las propiedades mecánicas superiores de los refractarios óxido-C [1,2] están relacionadas con la deformación inelástica (o flexibilidad) otorgada por la presencia de grafito [3]. Este comportamiento le permite al ladrillo acomodar el esfuerzo aplicado mediante cierta 'fluencia', incrementando la deformación de fractura. Desde este punto de vista, además del esfuerzo a la fractura, comúnmente usado como propiedad para evaluar mecánicamente este tipo de materiales [1, 4] (módulo de rotura MOR, resistencia a la compresión CCS, módulo de rotura en caliente HMOR), la deformación en esta condición es un dato igualmente importante. Las curvas esfuerzo-deformación son una fuente adecuada para acceder a esta información y pueden ser obtenidas en diferentes condiciones de temperatura y atmósfera [5-11]. Además, a partir de esas curvas pueden calcularse parámetros mecánicos indispensables para la obtención de ecuaciones constitutivas requeridas en la actualidad para alimentar los programas de elementos finitos empleados en el cálculo estructural. Junto con el análisis de los mecanismos de deformación y fractura, las relaciones esfuerzo-deformación son útiles para mejorar el diseño del material.

En la industria siderúrgica, principal consumidora de materiales refractarios, la fabricación del acero requiere condiciones químicas, térmicas y mecánicas que exigen contar con materiales de alto desempeño, capaces de soportar la acción del baño de acero y de la escoria. A pesar del incremento en el precio de la bauxita, el uso de ladrillos refractarios $\mathrm{Al}_{2} \mathrm{O}_{3}-\mathrm{MgO}-\mathrm{C}$ (AMC) en las plantas siderúrgicas mantiene niveles elevados de consumo debido a sus excelentes propiedades en relación a las condiciones de uso (elevadas resistencias al 'spalling' térmico y al ataque de escorias y excelentes propiedades mecánicas en caliente) [13-18]. Los refractarios AMC muestran mejor desempeño en instalaciones de fondos y paredes de cucharas de producción de acero respecto a los convencionales de alta alúmina o dolomíticos. Sin embargo, las condiciones en servicio cada vez más severas (altas temperaturas, entornos químicamente muy agresivos y solicitación mecánicas) conducen inevitablemente 
a la degradación de estos refractarios. Por esta razón, y con el objetivo de evaluar el desempeño en servicio y establecer criterios de diseño del material, el conocimiento de las propiedades mecánicas a alta temperatura es indispensable. La mejora de estos refractarios tiene como consecuencia el aumento de su vida útil y la disminución de costos e impacto medioambiental.

En este trabajo se presenta una metodología implementada en el Laboratorio de Materiales Estructurales de la División Cerámicos del INTEMA (Argentina) [19, 20], para evaluar mecánicamente refractarios óxido- $\mathrm{C}$ en condiciones de alta temperatura y atmósfera controlada, con vistas a abordar estudios científico-tecnológicos y a desarrollar modelos de comportamiento más precisos. En este trabajo se presenta como ejemplo de aplicación de la metodología la evaluación de ladrillos refractarios comerciales $\mathrm{Al}_{2} \mathrm{O}_{3}-\mathrm{MgO}-\mathrm{C}$ de uso en cucharas de acería [21].

\section{METODOLOGIA PARA LA DETERMINACION DE CURVAS ESFUERZO-DEFORMACION DE REFRACTA- RIOS OXIDO-C A ALTA TEMPERATURA Y ATMOSFE- RA CONTROLADA}

La metodología diseñada e implementada en el Laboratorio para la evaluación mecánica en caliente de materiales refractarios [20] se basa en la posibilidad de medir en forma directa la deformación axial de probetas sometidas a compresión. En el caso de materiales susceptibles a reaccionar con la atmósfera, se incluye un sistema para su modificación y control. La metodología que se describe en este trabajo se refiere a la utilizada para la obtención de curvas esfuerzodeformación con carga monotónica creciente, aunque los dispositivos también pueden emplearse para otro tipo de determinaciones (ensayos carga-descarga, curvas de creep, ensayos de relajación). El equipamiento básico que permite la ejecución de este tipo de ensayos está constituido por una máquina universal de ensayos mecánicos servohidráulica Instron (modelo 8501) a la que se acopla un horno eléctrico de cámara.

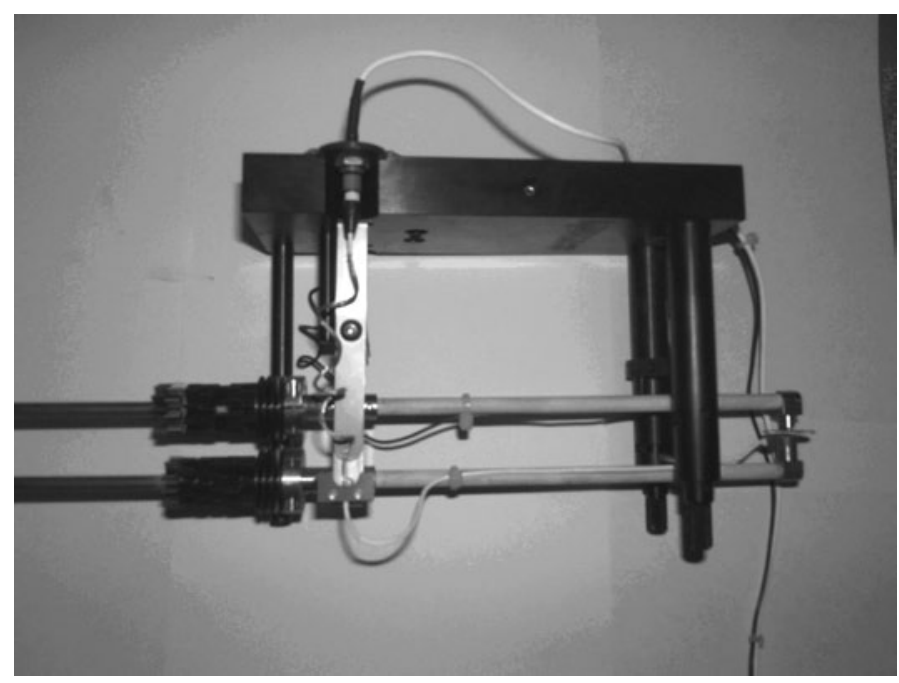

(a)
La medida directa de la deformación en estas condiciones no es trivial, y representa una seria limitación en la evaluación mecánica en caliente de materiales cerámicos en general. La extensometría de contacto, basada en el uso de extensores mecánicos en contacto con la probeta que reproducen su deformación y la transfieren a un transductor externo, fue seleccionada como técnica de medida dado que resulta en determinaciones más precisas que las alcanzadas por otras metodologías de uso común como la dilatometría diferencial [22]. Esto se debe a que la medida se realiza sobre la probeta, no resultando afectada por la deformación del tren de carga, y el uso de extensores largos permite la medida precisa de las pequeñas deformaciones que ocurren en el material refractario. Además, debido a la configuración del extensómetro es posible aislarlo del calor que disipa el horno en el que se realizan los ensayos, garantizando la estabilidad térmica del mismo durante el ensayo y la exactitud de las medidas. Aún así, el uso de extensómetros mecánicos, tiene asociadas ciertas dificultades experimentales, que requieren especial atención para garantizar la exactitud de la medida. Por ejemplo, la presión de contacto para evitar el deslizamiento de los extensores sobre la probeta es un punto clave en la operación de estos dispositivos. En este caso, se implementó el uso de dos tipos de extensómetros para alta temperatura, de acuerdo al rango de deformación a ser medido [20].

Por otra parte, la aplicación de carga compresiva suele ser común en la evaluación mecánica de refractarios, dada las complicaciones asociadas a la tracción en materiales frágiles y al hecho de que en el servicio de estos materiales suele predominar este tipo de solicitación. La utilización de separadores entre las probetas y las barras que aplican la carga, usualmente de un material cerámico denso, previene de posibles reacciones entre la probeta y las barras favorecidas por la compresión en condiciones de alta temperatura.

Se seleccionó la geometría cilíndrica para las probetas en base a las ventajas de su uso en ensayos de compresión respecto a otras geometrías [23] y por las menores dificultades de preparación, incluyendo la minimización del

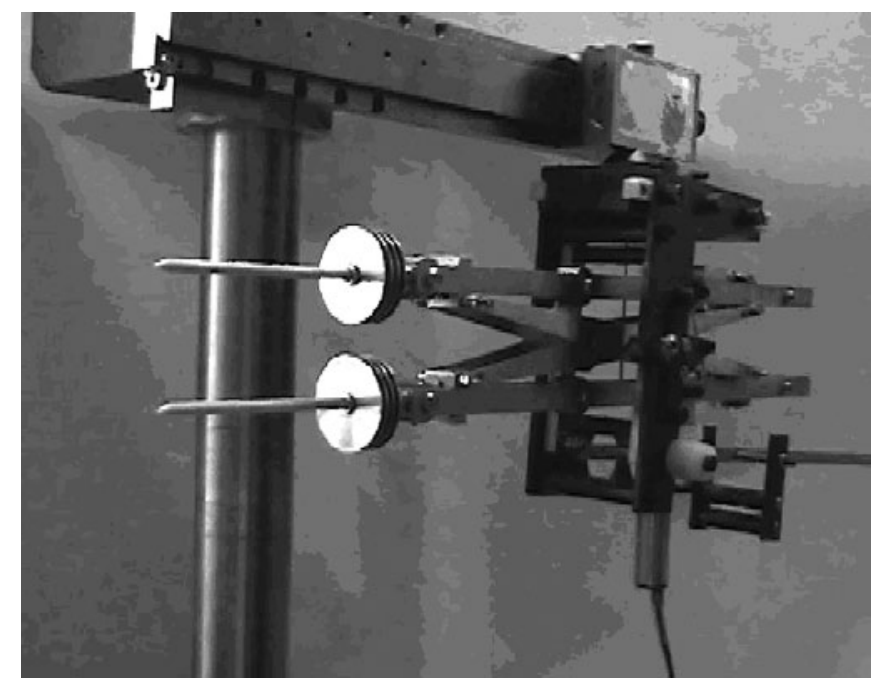

(b)

Figura 1. Extensómetros: (a) capacitivo; (b) de tijeras. 
mecanizado, que sólo es requerido en las caras planas. El tamaño de los cilindros se definió teniendo en cuentas varias cuestiones [20] que se discuten más adelante.

Finalmente, la disponibilidad de un horno eléctrico estándar, limitó la generación y control de atmósfera al desplazamiento por flujo de una corriente gaseosa dentro de una cámara cerámica introducida en el horno, una práctica común para este tipo de requerimiento.

Sobre la base de estas condiciones, se requirió la implementación de varias etapas de construcción, modificación, puesta a punto y calibración de los dispositivos utilizados [20], que se describen a continuación. Durante esta etapa se utilizaron ladrillos comerciales de $\mathrm{MgO}-\mathrm{C}$ de diferentes características.

\subsection{Medición de la deformación axial: extensómetros capacitivo y de tijeras}

Para medir la deformación durante los ensayos para la determinación de curvas esfuerzo-deformación, se cuenta con dos tipos de extensómetros mecánicos, de acuerdo al rango de variación dimensional que presente el material (Fig. 1). Para pequeñas variaciones $<0,1 \mathrm{~mm}$ como las que suelen presentar los ladrillos refractarios de sílice, de alta alúmina o básicos, se emplea un extensómetro capacitivo comercial (Instron). Para rangos de variación mayores a este límite, hasta el orden del milímetro, como la que exhiben los concretos refractarios, se utiliza un extensómetro de tijeras de diseño y construcción propios.

El extensómetro capacitivo (Fig. 1a) es un dispositivo comercial marca Instron que, junto con el calibrador (Fig. 2), son accesorios originales de la máquina universal de ensayos mecánicos (Instron 8501) con la que cuenta el Laboratorio. Los manuales con especificaciones e indicaciones para su armado, montaje y calibración/verificación, posicionamiento sobre la probeta y recomendaciones para su óptimo funcionamiento se basan en la norma ASTM E83 [24]. Este instrumento consta de dos extensores de $\mathrm{SiC}$, lo cual le permite operar a temperaturas hasta $1600^{\circ} \mathrm{C}$. La variación dimensional de la probeta durante el ensayo resulta en un movimiento de los brazos cerámicos del extensómetro que se transmite al transductor capacitivo el cual actúa variando la distancia entre el sensor del transductor y el plato de referencia. El transductor capacitivo está conectado a un amplificador-transductor que convierte la capacitancia en una señal eléctrica, proporcional a la variación dimensional de la probeta. De acuerdo al manual de especificaciones [25], este extensómetro tiene las siguientes características: precisión $\pm 0,6 \mu \mathrm{m}$ (equivalente a $\pm 24 \mu \varepsilon$ a $25 \mathrm{~mm}$ de 'gauge length'); resolución $0,2 \mu \mathrm{m}$; recorrido $\pm 0,1 \mathrm{~mm}$; sobrerecorrido $120 \%$ de deflexión a fondo de escala; fuerza de contacto $\sim 35 \mathrm{~g}$ por borde agudo (ajustable de 0 a $100 \mathrm{~g}$ ); 'gauge length' $25 \mathrm{~mm}$. El uso de este extensómetro requiere de una calibración previa del LVDT que se realiza con ayuda del calibrador (Fig. 2), el cual permite obtener desplazamientos micrométricos con mucha precisión $(0,001 \mathrm{~mm})$.

El extensómetro de tijeras (Fig. 1b) tiene un mecanismo de operación similar al capacitivo, con la diferencia de que los extensores, en este caso de alúmina, se mantienen siempre paralelos durante su movimiento, con las ventajas asociadas en la transmisión de la medida hacia el sensor. Previo a su uso, sus varios componentes se analizaron detalladamente en cuanto a su funcionamiento y condiciones óptimas de operación [20]. Durante el análisis de su funcionamiento, se estableció que los errores de medición del sistema se originan en pérdidas mecánicas, requiriéndose un ajuste adecuado de los varios tornillos y prisioneros que lo constituyen, y se establecieron las siguientes especificaciones [20]: precisión \pm $30 \mu \mathrm{m}$ (equivalente a $\pm 860 \mu \varepsilon$ a $35 \mathrm{~mm}$ de 'gauge length'); resolución 0,1 $\mu \mathrm{m}$; recorrido $+5 \mathrm{~mm} /-7,4 \mathrm{~mm}$ (modificable); sobrerecorrido $110 \%$ de deflexión a fondo de escala; 'gauge length' $35 \mathrm{~mm}$ (modificable); recorrido de precarga $1 \mathrm{~mm}$ (equivalente a $50 \mathrm{~g}$ en borde de varilla-modificable); máxima temperatura de operación: $1600{ }^{\circ} \mathrm{C}$. Para fijar la carga de contacto $(\sim 50 \mathrm{~g})$ se consideró la recomendación de los fabricantes del extensómetro capacitivo. El análisis del error que arroja el extensómetro en diferentes rangos de medición permite establecer que este dispositivo puede utilizarse aceptablemente en el rango de $1 \mathrm{~mm}$ para deformaciones de aproximadamente $2 \%$ [20]. El LVDT del extensómetro de tijeras es autocalibrado independientemente de la parte mecánica, por lo cual previo a su uso se requiere de calibración y verificación en función de los ajustes de las partes mecánicas con ayuda del calibrador (Fig. 2) [20].

\subsection{Aplicación de la carga y geometría de probeta}

El sistema servohidráulico de la máquina de ensayos mecánicos permite un control muy preciso del movimiento del actuador, del orden del $\mu \mathrm{m} / \mathrm{min}$. Además, los ensayos pueden realizarse por control de carga, desplazamiento o deformación. La carga compresiva se aplica mediante dos barras de mullita/alúmina de alta densidad de $60 \mathrm{~mm}$ de diámetro, que no forman parte del equipamiento original sino que constituyen parte de las adaptaciones realizadas en el sistema para la evaluación de materiales refractarios. Las mismas se ajustan al cabezal y al actuador mediante adaptadores de diseño y construcción propia, refrigerados por agua. A su vez, la máquina dispone originalmente de anillos de calibración que permiten la alineación axial del sistema de carga con una precisión de 0,01 $\mathrm{mm} / \mathrm{m}$. Dado que la carga compresiva y la alta temperatura favorecen las reacciones entre la probeta y las barras cerámicas, se colocan entre ambas discos separadores (aproximadamente $1 \mathrm{~cm}$ de altura y 50-60 $\mathrm{mm}$ de diámetro), generalmente de alúmina.

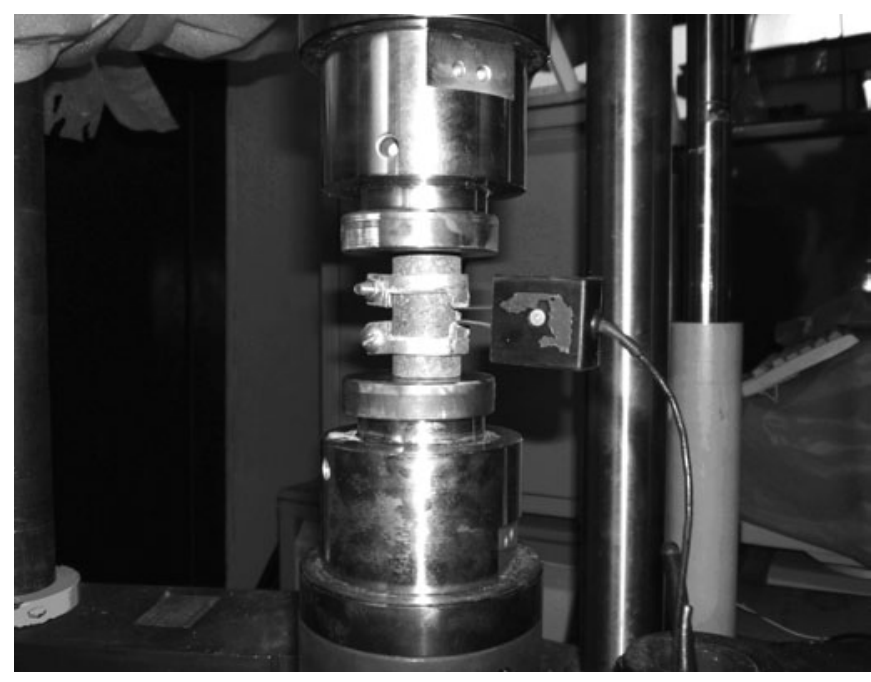

Figura 2. Calibrador (Instron). 
El ensayo para la obtención de curvas esfuerzodeformación con carga monotónica creciente, se realiza en control por deslazamiento ya que el uso de una velocidad de deformación constante no resultó en un control estable del ensayo. Para establecer la velocidad de desplazamiento debe tenerse en cuenta que el objetivo del ensayo es evaluar la respuesta esfuerzo-deformación evitando introducir efectos temporales como el de impacto en el caso de trabajar con altas velocidades o el de crecimiento subcrítico de fisuras en el caso de utilizar velocidades demasiado pequeñas. Para establecer una condición adecuada se realizó un análisis de las curvas esfuerzo-deformación en el rango 0,02 y 0,3 mm/ min sobre materiales comerciales $\mathrm{MgO}-\mathrm{C}$ [20]. Se encontró que un valor alrededor de $0,1 \mathrm{~mm} / \mathrm{min}$ permite obtener registros representativos y comparables, sin efectos temporales sobre el comportamiento esfuerzo-deformación en este tipo de materiales [20]. Sin embargo, este valor es orientativo y puede requerir de un ajuste para el caso de materiales con propiedades diferentes.

Las dimensiones de los cilindros empleados como probetas para los ensayos, $27 \mathrm{~mm}$ de diámetro y $50 \mathrm{~mm}$ de altura, se establecieron teniendo en cuenta varios aspectos [20], que se discuten a continuación:

(a) efecto volumétrico, ligado a la representatividad microestructural de la probeta; un comportamiento representativo del material sólo se puede obtener cargando un volumen suficientemente grande en términos microestructurales. En la norma ASTM 133-94 [26] de ensayos en compresión de materiales refractarios se establece que la menor dimensión de la probeta debe ser por lo menos cuatro veces mayor al tamaño del agregado más grande. Para establecer una referencia, y considerando que en general los agregados de los materiales refractarios óxido-C son menores a 5-6 mm, se consideró que los cilindros deberían tener, como mínimo, un diámetro de $24 \mathrm{~mm}$.

(b) efecto estructural, relacionado al campo de esfuerzos que se desarrolla en la probeta, el cual está afectado por los efectos de fricción que se generan en la zona de contacto con el sistema de aplicación de la carga. Este fenómeno restringe el desplazamiento libre del material de las caras de apoyo, lo cual genera esfuerzos radiales y tangenciales en la proximidad de los bordes, afectando la deformación de la probeta. Este efecto se minimiza comúnmente con el empleo de un lubricante entre la probeta y las barras; lamentablemente, esta estrategia no puede usarse a alta temperatura. Otra posibilidad es emplear probetas con una relación de altura/diámetro elevada, tal que la zona afectada por los efectos de fricción sea pequeña con respecto al volumen total. Sin embargo, una relación de aspecto muy grande puede causar el pandeo del cilindro durante la compresión; se ha sugerido una relación altura/diámetro mayor que 2 para probetas cilíndricas sometidas a compresión [23].

(c) limitaciones de la carga máxima del sistema $(50 \mathrm{kN})$, dada por la capacidad de la celda de carga $(100 \mathrm{kN})$ y de las barras cerámicas. (d) restricciones de las dimensiones del horno, del tren de carga y del sistema de control de atmósfera.

(e) las posibilidades de fabricación y mecanizado en el Laboratorio; los cilindros se obtienen por corte de los ladrillos utilizando discos y brocas diamantados, y posterior mecanizado de las caras planas con muelas de diamante. El diámetro de probeta, que se fijó finalmente en $27 \mathrm{~mm}$, tuvo en cuenta la disponibilidad de brocas de diámetro interno estándar.

En la preparación de las probetas, la dirección de corte se establece de acuerdo a los objetivos de estudio. El lubricante y refrigerante utilizado (agua, aceite, kerosene, o ninguno) depende de las características del material óxido-C y su tendencia a la hidratación. El equipamiento y condiciones para llevar a cabo estos procedimientos requirieron de una puesta a punto con vistas a establecer las condiciones óptimas para obtener probetas de la calidad y tolerancia dimensional adecuadas (planoparalelismo $<0,2 \mathrm{~mm}$ ) [20]. Previamente a los ensayos mecánicos, las probetas se secan a $100{ }^{\circ} \mathrm{C}$ durante $24 \mathrm{~h}$ en estufa y luego se enfrían en vacío dentro de un desecador para reducir la incorporación de la humedad ambiental hasta ser ensayadas.

\subsection{Control de la atmósfera}

Los materiales refractarios óxido-C son muy susceptibles a reaccionar con el $\mathrm{O}_{2}$ del aire por encima de los $500^{\circ} \mathrm{C}$, en particular el grafito. La consecuente decarburización modifica su respuesta mecánica por la pérdida de cohesión entre los agregados y la reducción de la sección resistente y del aporte de características que le imparte el grafito como la flexibilidad. Como consecuencia, para evaluar la respuesta mecánica del material, resulta conveniente hacerlo en condiciones en las cuales se minimice el solapamiento de la pérdida de grafito por oxidación [9], para lo cual se requiere evitar la presencia del agente oxidante en la atmósfera del horno que circunda la probeta. Sin embargo, el grafito también puede ser consumido en reacciones con los propios componentes del sistema, como es el caso típico de la reducción carbotermal de la magnesia

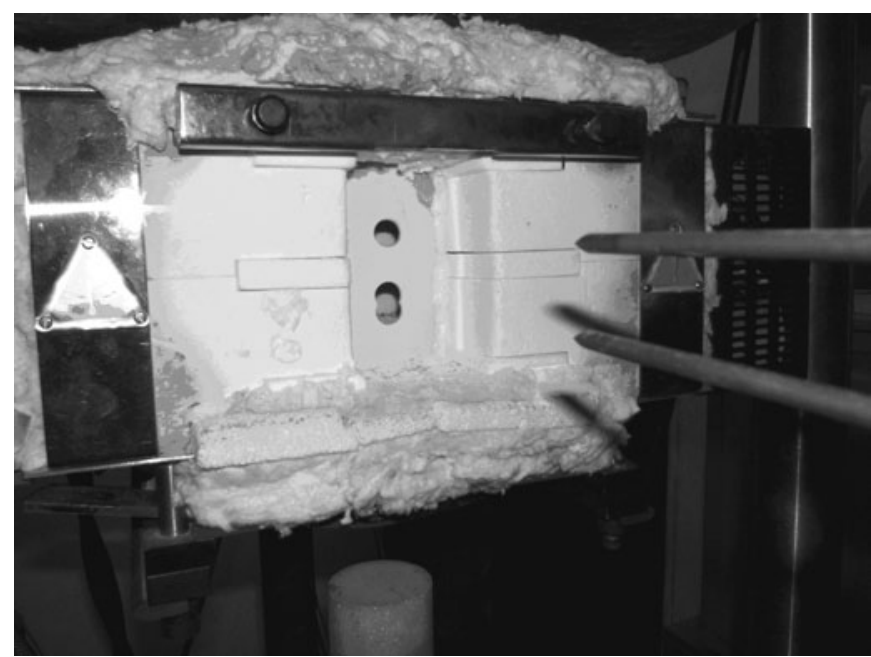

Figura 3. Sistema de atmósfera controlada: vista frontal de la cámara con los orificios para el ingreso del extensómetro. 
$\left(\mathrm{MgO}_{(\mathrm{s})}+\mathrm{C}_{(\mathrm{s})} \rightarrow \mathrm{Mg}_{(\mathrm{g})}+\mathrm{CO}_{(\mathrm{g})}\right)$, que ocurre en el rango entre $1200 \mathrm{y}$ $1860^{\circ} \mathrm{C}$; esta reacción se llevará a cabo independientemente de la atmósfera circundante (aunque, obviamente, su evolución depende de las presiones parciales de $\left.\mathrm{CO}, \mathrm{Mg} \mathrm{y} \mathrm{O}_{2}[10]\right)$ por lo cual los resultados de la evaluación mecánica dependerán de la atmósfera utilizada $[1,4]$.

El horno disponible para estos ensayos (marca SFL Severn Furnance Ld) está dividido en dos zonas de calentamiento con sendos programadores/controladores (Eurotherm), cada una de las cuales consta de 6 elementos calefactores de $\mathrm{MoSi}_{2}$ en disposición radial, de modo que la cámara se reduce a un volumen utilizable de $80 \mathrm{~mm}$ de diámetro, aproximadamente. El horno tiene un agujero superior e inferior por donde ingresan las barras de aplicación de la carga. Para satisfacer el requerimiento de control de la atmósfera empleando este horno y el tren de carga descripto en la sección anterior, se diseñó y construyó un sistema que se basa en el desplazamiento por pasaje de una corriente gaseosa, generalmente $\mathrm{N}_{2}$, dentro de un tubo de material cerámico (mufla) que se introduce en el interior del horno (Fig. 3). El caudal de $\mathrm{N}_{2}$ que ingresa a la mufla, además de desplazar al agente oxidante, en este caso $\mathrm{O}_{2}$, reduce su concentración (efecto de dilución). En estas condiciones, el pasaje continuo de $\mathrm{N}_{2}$ asegura una sobrepresión dentro de la cámara impidiendo el ingreso de aire a presión atmosférica.

La mufla empleada con este objetivo debe cumplir los siguientes requisitos: a) no interferir en el tren de aplicación de la carga compresiva permitiendo el libre desplazamiento del actuador, b) permitir el ingreso, posicionamiento y libre desplazamiento del extensómetro, c) no degradarse químicamente, ni deformarse o romperse en las condiciones térmicas y atmosféricas del ensayo, d) no incrementar excesivamente la inercia térmica del horno. En base a estas restricciones se seleccionó como mufla un tubo de alúmina sinterizada de alta densidad $(99,9 \%)$ de 70 y $76 \mathrm{~mm}$ de diámetros interno y externo, respectivamente y $220 \mathrm{~mm}$ de altura, con orificios para la entrada de los termopares de control del horno (parte posterior) y el canal de alimentación del gas (tubo de alúmina de $10 \mathrm{~mm}$ diámetro y $300 \mathrm{~mm}$ largo) y extensores del extensómetro (parte anterior, donde se encuentra la puerta del horno). Además de generar una cámara cuasi-estanca minimizando el caudal de $\mathrm{N}_{2}$ requerido, la mufla permite aislar los elementos calefactores de $\mathrm{MoSi}_{2}$ de la corriente del $\mathrm{N}_{2^{\prime}}$ lo cual tiende a eliminar la capa protectora de $\mathrm{SiO}_{2}$ que se forma en aire [27]. El caudal del nitrógeno necesario para reducir la decarburización a un nivel aceptable (pérdida de peso de $\approx 1 \%$ y ausencia de decoloración hacia el interior de la probeta detectada por inspección visual, a $1000^{\circ} \mathrm{C}$ ), teniendo en cuenta un compromiso entre el grado de oxidación y el consumo de gas (por cuestiones de costo), requirió de un estudio específico [20]. Como resultado se estableció un valor de $5 \mathrm{l} / \mathrm{min}$, con un período previo de purga de la mufla, para un flujo de $\mathrm{N}_{2}$ de pureza 99,996\%.

Para minimizar el efecto de la disminución de la cámara del horno a consecuencia de la presencia de la mufla se aumentaron el tamaño de la cámara (alejando los elementos calefactores) y de las aberturas de techo y se construyó un soporte en el piso del horno para sostener la mufla. Estas modificaciones tuvieron un efecto significativo sobre la eficiencia y la temperatura máxima del horno [20]: las temperaturas y la velocidad de calentamiento máximas se redujeron de $1600^{\circ} \mathrm{C}$ a $1375^{\circ} \mathrm{C}$ y de $17^{\circ} \mathrm{C} / \mathrm{min}$ a $10^{\circ} \mathrm{C} / \mathrm{min}$.
Mediante el uso de medidas de la temperatura sobre la probeta se fijaron las condiciones óptimas de calentamiento y estabilización teniendo en cuenta la condición térmica del horno y lo sugerido en las normas [20]: $10^{\circ} \mathrm{C} / \mathrm{min}$ hasta 1100 ${ }^{\circ} \mathrm{C}, 5{ }^{\circ} \mathrm{C} /$ min entre 1100 y $1400{ }^{\circ} \mathrm{C}$ y 15 min de estabilización a la temperatura final previo al ensayo.

\subsection{Ensayo para la determinación de las curvas esfuerzo- deformación}

La etapa de puesta a punto de este ensayo se realizó sobre probetas de ladrillos comerciales de $\mathrm{MgO}-\mathrm{C}$ de diferentes calidades, desde temperatura ambiente hasta $1400^{\circ} \mathrm{C}$ [20]. Se utiliza el equipamiento y condiciones presentadas en los puntos anteriores, las cuales se encuentran descriptas en un protocolo [28] que explica en detalle cómo llevar a cabo todos los procedimientos implicados, desde el encendido de la máquina hasta la obtención de la curva esfuerzo-deformación. Básicamente el ensayo consiste de las siguientes etapas:

1) posicionamiento de la probeta y el extensómetro (previamente calibrado) dentro del horno incluyendo la disposición de la mufla,

2) calentamiento de la probeta hasta la temperatura del ensayo y estabilización; durante esta etapa se aplica una pequeña pre-carga compresiva para asegurar el contacto del sistema de aplicación de la carga, la probeta y el extensómetro,

3) aplicación de la carga a velocidad de desplazamiento constante hasta rotura de la probeta, para la determinación de la curva esfuerzo-deformación,

4) enfriamiento de la probeta.

Esta última etapa no es trivial porque pueden seguir ocurriendo modificaciones sobre la probeta que alteran el análisis post-ensayo, que es imprescindible para la evaluación de mecanismos de deformación y fractura. El protocolo también explica el uso de los programas para el diseño y control de los ensayos y adquisición de datos, que en gran parte son suministrados por Instron, aunque también se requiere de otros programas para el procesamiento de los datos post-ensayo.

Las curvas esfuerzo-deformación obtenidas empleando el protocolo de ensayo en las mismas condiciones experimentales sobre diferentes probetas extraídas de un ladrillo comercial presentan una cierta variabilidad atribuible a la heterogeneidad que presenta un material refractario de este origen, como puede observarse en la Fig. 4 para ladrillos comerciales de $\mathrm{MgO}-\mathrm{C}$ ligados con alquitrán y resina, ensayados a temperatura ambiente y a $1400^{\circ} \mathrm{C}$, respectivamente.

A partir de las curvas esfuerzo-deformación se estudia el comportamiento mecánico del material (elástico, inelástico, plástico, con presencia de ablandamiento, etc.) y se determinan parámetros mecánicos como el módulo de elasticidad, la resistencia mecánica, la deformación de fractura, el límite elástico. En algunos casos pueden emplearse las definiciones convencionales de los parámetros o bien establecer otras más adecuadas al comportamiento de materiales complejos como los refractarios óxido-C [20]. De acuerdo a los resultados de la puesta a punto de la metodología sobre materiales de origen comercial, se han establecido como errores máximos de los parámetros mecánicos: $\pm 25 \%$ para el módulo de elasticidad y $\pm 20 \%$ para la resistencia mecánica. 
Además de la obtención de la curva esfuerzo-deformación y de los parámetros mecánicos, este ensayo se complementa con información adicional que puede ser usada para estudios básicos de mecanismos de deformación y fractura. Como parte de la rutina del ensayo se determina la variación de peso que sufre la probeta y se registran características de la falla del material, como la presencia o ausencia de ruido. Además, luego del ensayo se inspecciona la probeta para determinar el tipo fractura, es decir, cantidad y orientación de las fisuras, si las mismas corren a través de la matriz, de la interfase con los agregados o de los agregados en sí mismos, para lo cual se observa a ojo desnudo o con lupa estereoscópica. En el caso del estudio de mecanismos, a esta información se le suma el análisis mineralógico por difracción de rayos $X$ (XRD), microestructural por microscopía óptica y electrónica de barrido con análisis químico por espectroscopía de energía dispersiva de rayos X (SEM/EDS), entre otras.

La metodología descripta puede requerir de la implementación de algunos ajustes en las condiciones experimentales en el caso de que los materiales a ser estudiados presenten propiedades significativamente diferentes a aquéllos sobre los cuales se basó la puesta a punto (MgO-C).

\section{EJEMPLO DE APLICACIÓN: MATERIALES REFRACTA- RIOS $\mathrm{AL}_{2} \mathrm{O}_{3}-\mathrm{MgO}-\mathrm{C}$}

La metodología para la determinación de curvas esfuerzodeformación se aplicó al estudio de ladrillos comerciales $\mathrm{Al}_{2} \mathrm{O}_{3}$ $\mathrm{MgO}-\mathrm{C}(\mathrm{AMC})$ de uso en cucharas de acería, en condiciones de atmósfera variables (aire y nitrógeno) y desde temperatura ambiente (RT) hasta $1260{ }^{\circ} \mathrm{C}$. Se presentan algunos de los resultados obtenidos en esta evaluación para uno de los materiales estudiados.

Desde un punto de vista mecánico, los ladrillos refractarios AMC pueden ser considerados como materiales heterogéneos constituidos por una fase discontinua de agregados de alúmina y/o magnesia de diferentes tamaños embebidos en una matriz continua que contiene un ligante hidrocarbonado (resina), granos finos de alúmina y magnesia, escamas de grafito y aditivos antioxidantes (metálicos $\mathrm{u}$ otros). La presencia de partículas antioxidantes de aluminio y silicio, entre otros, incrementan el rendimiento mecánico de los ladrillos a alta temperatura por la posibilidad de formación de carburo ó nitruro de aluminio $\left(\mathrm{Al}_{4} \mathrm{C}_{3}\right.$ ó $\left.\mathrm{AlN}\right)$, estables a temperaturas inferiores a $1200{ }^{\circ} \mathrm{C}$, y de carburo de silicio $(\mathrm{SiC})$ y espinela $\left(\mathrm{MgO}-\mathrm{Al}_{2} \mathrm{O}_{3}\right)$ a más altas temperaturas [1, 10, 29, 30]. La evolución de estas fases depende fuertemente de la atmósfera circundante. La formación de los carburos y nitruros de aluminio (por reacción del $\mathrm{Al}$ con el carbono de la descomposición del ligante o el grafito, en el primer caso, y con el nitrógeno atmosférico en el segundo) se favorece a bajas presiones parciales de oxígeno ya que tienden a descomponerse fácilmente en $\mathrm{Al}_{2} \mathrm{O}_{3}[10,30]$; la presencia de $\mathrm{Al}_{4} \mathrm{C}_{3}$ se ha detectado aún en atmósfera de $\mathrm{Ar}[10,29]$. El efecto positivo de estas fases sobre las propiedades mecánicas se ha atribuido principalmente a su morfología (tipo placa o esqueleto en el caso del carburo de aluminio) y a la formación de una red de cristales que favorece la unión de la estructura ('binding effect') $[10,29]$.

\subsection{Materiales}

De acuerdo a la hoja técnica del proveedor, el ladrillo AMC presentado como ejemplo está constituido por alúmina tabular (66\% en peso de $\mathrm{Al}_{2} \mathrm{O}_{3}$ ), magnesia sinterizada (32 $\%$ en peso de $\mathrm{MgO}$ ), grafito, resina como ligante y aditivos metálicos como antioxidantes. Esta información se confirmó por análisis cualitativo de fases por XRD y SEM/EDS, a partir de lo cual también se estableció la naturaleza del aditivo metálico: aluminio. En la Fig. 5 se muestra la superficie pulida del material original en la que se observan varios tipos de agregados, de diferente coloración. A partir del análisis por SEM/EDS (Fig. 6) se confirmó la presencia de agregados de alúmina tabular (blanca), alúmina electrofundida marrón (beige claro y marrón oscuro, debido a la presencia de Ti) y de magnesia sinterizada (beige oscuro). La forma angulosa de los agregados es propia de una molienda; el tamaño medio de los agregados de alúmina es mayor al de las partículas de magnesia.
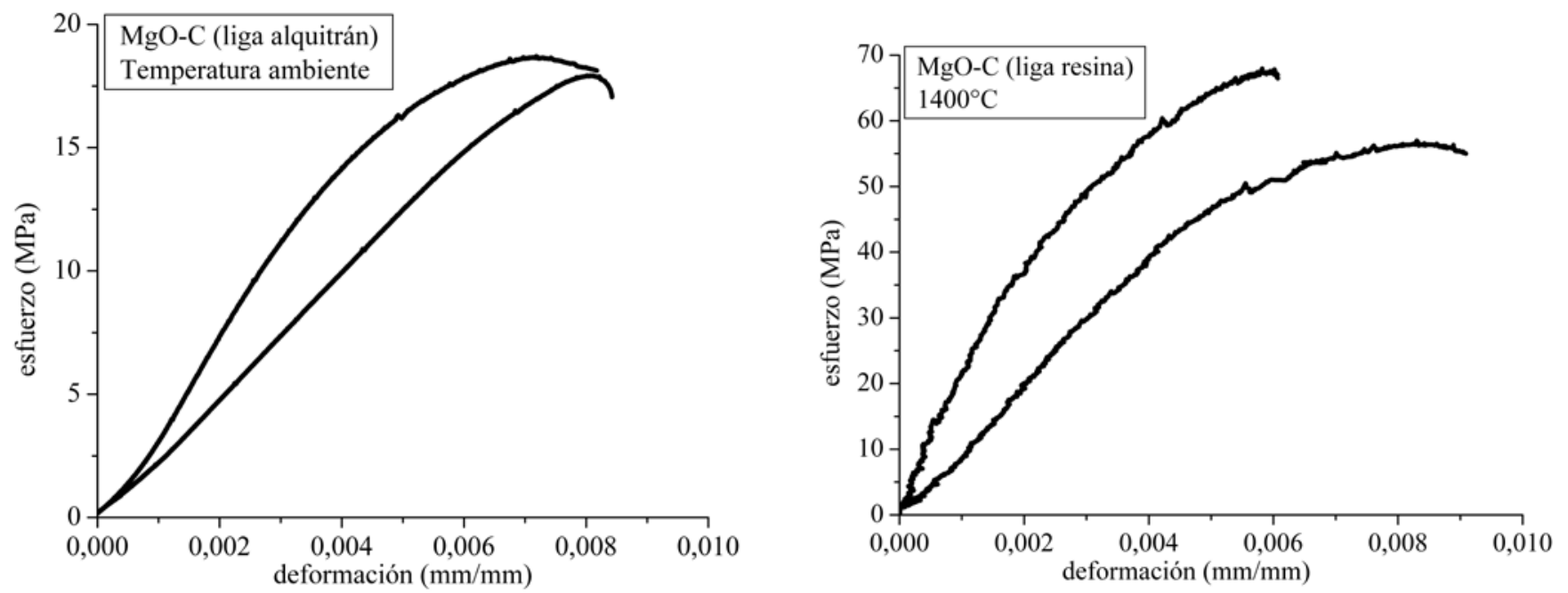

Figura 4. Curvas esfuerzo-deformación para ladrillos comerciales de MgO-C ligados con alquitrán y resina, ensayados a temperatura ambiente y a $1400{ }^{\circ} \mathrm{C}$, respectivamente. 


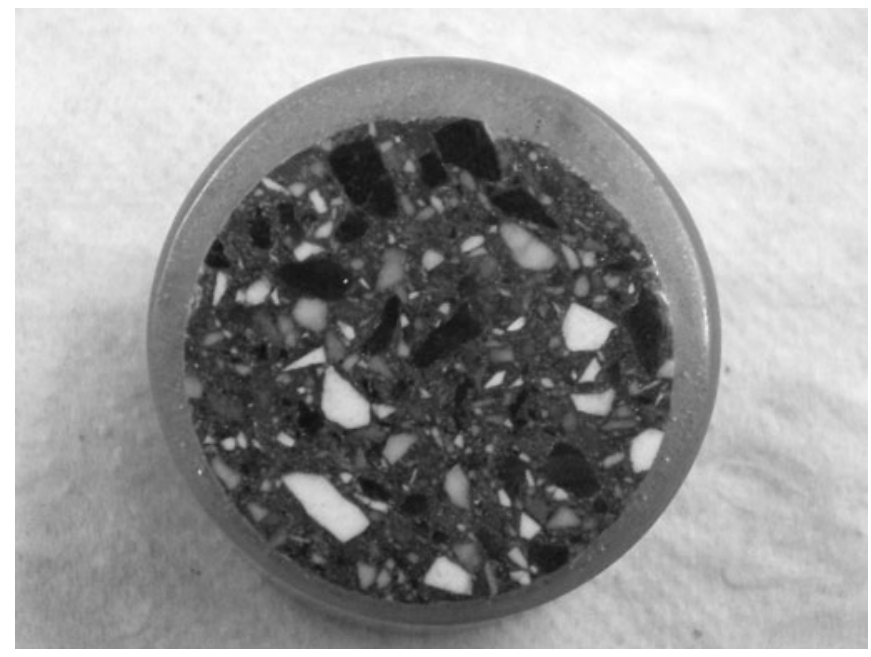

Figura 5. Superficie del material AMC.

Siguiendo un protocolo interno basado en la norma DIN EN 993-1(DIN 51056) [31], se determinaron los valores de densidad global $\left(\rho_{\mathrm{b}}\right)$ y porosidad aparente $\left(\pi_{\mathrm{ap}}\right)$ en kerosene a $37^{\circ} \mathrm{C}$, por duplicado, obteniéndose los siguientes valores: $2,98 \pm 0,01 \mathrm{~g} / \mathrm{cm}^{3}$ y 7,8 $\pm 0,5 \%$, respectivamente. Por análisis térmico diferencial y termogravimétrico (DTA/TGA) en aire hasta $1400{ }^{\circ} \mathrm{C}\left(10^{\circ} \mathrm{C} / \mathrm{min}\right)$ se registraron picos exotérmicos asociados a pérdidas de peso. Estos se atribuyen a la transformaciones de la resina (DTA: $400-530{ }^{\circ} \mathrm{C} / \mathrm{TGA}:-5,5 \%$ en peso) y la oxidación del grafito (DTA: $640-900{ }^{\circ} \mathrm{C} / \mathrm{TGA}$ : $-3,4 \%$ en peso), en acuerdo con el contenido total de C (8\% en peso según la hoja técnica). A mayores temperaturas (1200$\left.1400{ }^{\circ} \mathrm{C}\right)$, se registró una alteración en el termograma de DTA, muy poco definida, que puede asociarse a la formación de espinela $\mathrm{MgAl}_{2} \mathrm{O}_{4}$ (obtenida por la reacción de $\mathrm{Al}_{4} \mathrm{C}_{3}$ ó $\mathrm{Al}_{2} \mathrm{O}_{3}$ con $\mathrm{MgO}$ ó $\mathrm{Mg}(\mathrm{g})$ ).

\subsection{Curvas esfuerzo-deformación y parámetros mecánicos}

En la Fig. 7 se grafican curvas esfuerzo-deformación del refractario AMC obtenidas a temperatura ambiente y a $1260^{\circ} \mathrm{C}$ en aire y atmósfera de $\mathrm{N}_{2}$ usando el extensómetro capacitivo y separadores de alúmina. En el caso de los ensayos a RT, se reemplazaron las barras de mullita/alúmina por platos y separadores de acero y en los ensayos a $1260^{\circ} \mathrm{C}$ en aire se eliminó el sistema de control de atmósfera. Los separadores utilizados funcionaron adecuadamente para este material $\mathrm{AMC}$, sin registrarse interacción con la probeta en la zona de contacto probeta/separador, ni tampoco entre las superficies de contacto separador/barras de aplicación de la carga.

En la Fig. 8 se observa el aspecto de las probetas luego de los ensayos a alta temperatura. En la probeta ensayada en aire se observa decoloración debido a la pérdida del grafito y de la estructura carbonosa no-grafitizable que resulta de la transformación de la resina. Luego del ensayo en nitrógeno, prevalece un núcleo oscuro con decoloración superficial, lo cual pone en evidencia la efectividad de la atmósfera utilizada en la minimización de los procesos oxidativos.

Se observó que en todas las probetas la fractura ocurrió principalmente a través de la matriz carbonosa, como también en la interfase agregado/matriz, tanto a temperatura ambiente

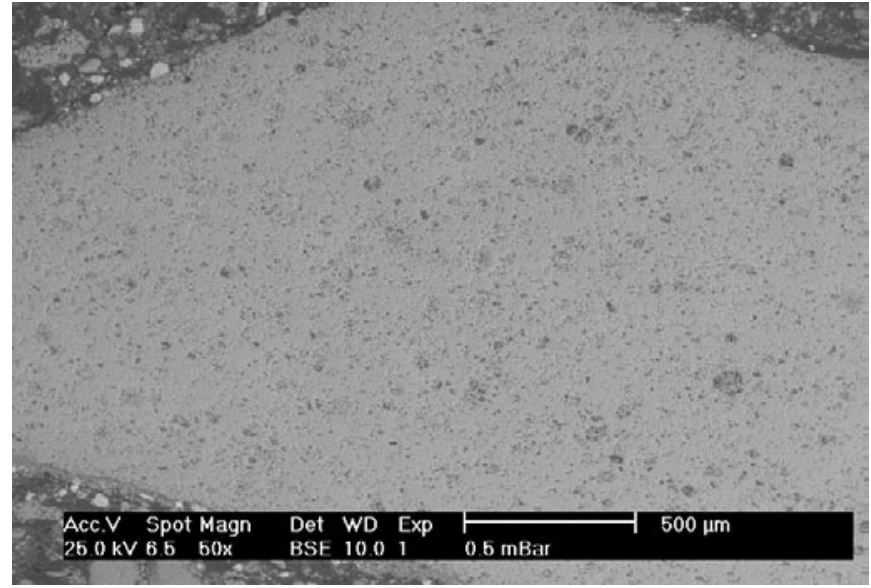

alúmina tabular

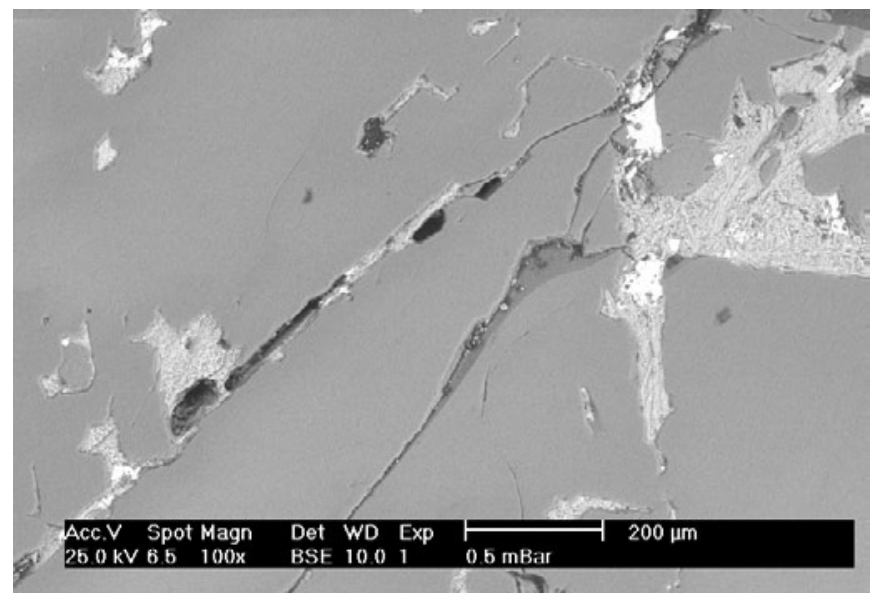

alúmina electrofundida marrón

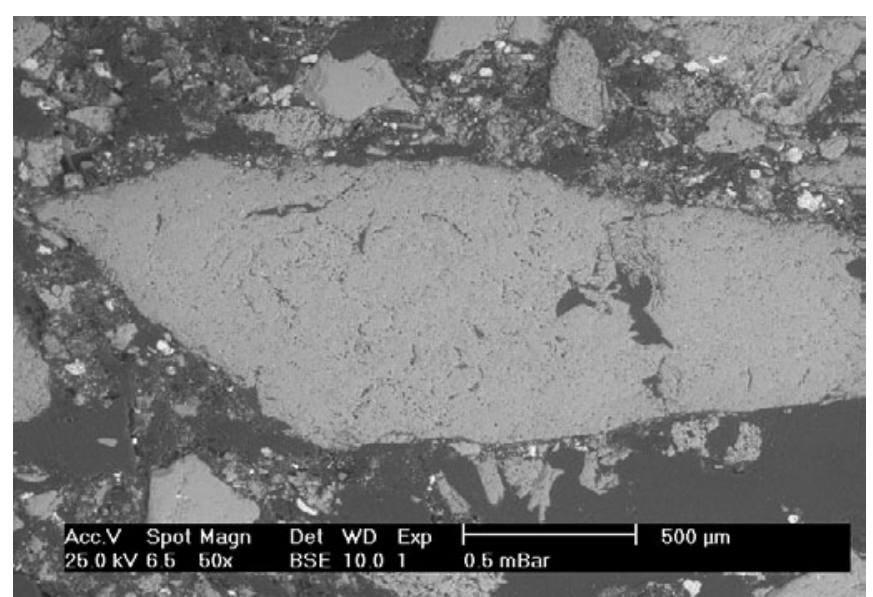

magnesia sinterizada

Figura 6. Imágenes de SEM de agregados del material AMC. 
TABLA I. VALORES DE LA DENSIDAD, POROSIDAD Y PARÁMETROS MECÁNICOS DE AMC

\begin{tabular}{|c|c|c|c|c|c|}
\hline & $\begin{array}{c}\boldsymbol{\rho}_{\mathrm{ap}} \\
\left(\mathbf{g} / \mathrm{cm}^{3}\right)\end{array}$ & $\mathbf{\Pi}_{\mathrm{ap}} \mathbf{( \% )}$ & $\begin{array}{c}\boldsymbol{\sigma}_{\mathrm{R}} \\
(\mathbf{M P a})\end{array}$ & $\varepsilon_{\mathrm{R}} \mathbf{( \% )}$ & $\begin{array}{c}\mathbf{E} \\
(\mathbf{G P a})\end{array}$ \\
\hline $\mathbf{R T}$ & 2,9 & 8 & 27 & 0,4 & 8,0 \\
\hline $\mathbf{1 2 6 0}^{\circ} \mathbf{C}$, aire & 2,6 & 28 & 11 & 1,1 & 0,9 \\
\hline $\mathbf{1 2 6 0}^{\circ} \mathbf{C}, \mathbf{N}_{2}$ & 2,8 & 18 & 19 & 1,0 & 2,0 \\
\hline
\end{tabular}

como a alta temperatura. Esto confirma el rol fundamental de la fase ligante en la respuesta mecánica del material de acuerdo con otros de composición similar [12, 20]. No se descarta que también haya ocurrido fractura intra-agregados, aunque en muy baja proporción.

En la Tabla I se reportan los valores de densidad global y densidad aparente de las probetas ensayadas a $1260{ }^{\circ} \mathrm{C}$; respecto al material original, los valores de densidad fueron más bajos, asociados a un mayor volumen de poros abiertos. El aumento de la porosidad fue menor en corriente de nitrógeno, en acuerdo con menores grados de avance de la oxidación del grafito y pirólisis del ligante (que aporta porosidad por la eliminación de volátiles y fisuras por las variaciones volumétricas que tienen lugar) [32].

El análisis por DRX sobre muestras en polvo de las probetas ensayadas a $1260^{\circ} \mathrm{C}$ mostró evidencias de formación de espinela en ambas condiciones de ensayo (aire y nitrógeno). La intensidad de los picos de espinela respecto a los correspondientes a los otros componentes del material, fue mayor en el material ensayado en aire que en el ensayado en nitrógeno, en acuerdo con lo reportado en la literatura [13]. No se identificó carbono como grafito en el material ensayado en aire, lo cual es consistente con la decoloración observada en las probetas ensayadas en estas condiciones. La formación de espinela está asociada a un incremento de volumen que puede generar fisuras que contribuyan al aumento de porosidad registrado en las probetas ensayadas a $1260^{\circ} \mathrm{C}$.

Las curvas esfuerzo-deformación indican un comportamiento cuasi-frágil en todas las condiciones. Para pequeñas deformaciones, las curvas son prácticamente lineales y luego se apartan de la linealidad hasta alcanzar la carga máxima. La no-linealidad es más marcada a elevada temperatura y en atmósfera de $\mathrm{N}_{2}$. Este comportamiento se puede atribuir a mecanismos de deformación irreversible, que ocurren a RT y a elevada temperatura, incluyendo [8, $12,33]$ : a) la propagación de fisuras y poros pre-existentes y originados durante el ensayo (microfisuración), especialmente aquéllos presentes en la matriz, b) el deslizamiento y repliegue de las escamas de grafito y c) la deformación plástica del ligante orgánico. En el ensayo a $1260{ }^{\circ} \mathrm{C}$ en aire, se favorece la oxidación de los productos de transformación de la resina y del grafito, por lo cual se espera que los mecanismos b) y c) no tengan un aporte significativo. Por el contrario, la microfisuración (a) se verá favorecida por el aumento de la porosidad y la formación de microfisuras (por la oxidación del grafito y la transformación de la resina); este hecho podría explicar el mayor desarrollo del comportamiento no-lineal en esta condición respecto a lo que ocurre a RT. Algo similar ocurre en los ensayos mecánicos en nitrógeno a $1260^{\circ} \mathrm{C}$. Sin embargo, la transformación térmica de la matriz se produce en menor grado en nitrógeno que en aire, lo cual aumenta la contribución de los mecanismos b) y c) y, por otro lado, la microfisuración se ve desfavorecida. Las curvas esfuerzodeformación muestran que el primer efecto domina sobre el segundo, teniendo en cuenta el mayor desarrollo de la respuesta no-lineal en esta condición. Además, a $1260^{\circ} \mathrm{C}$ ya podrían estar activos mecanismos visco-plásticos [12] debido a la presencia de fase vítrea, fundamentalmente en la matriz

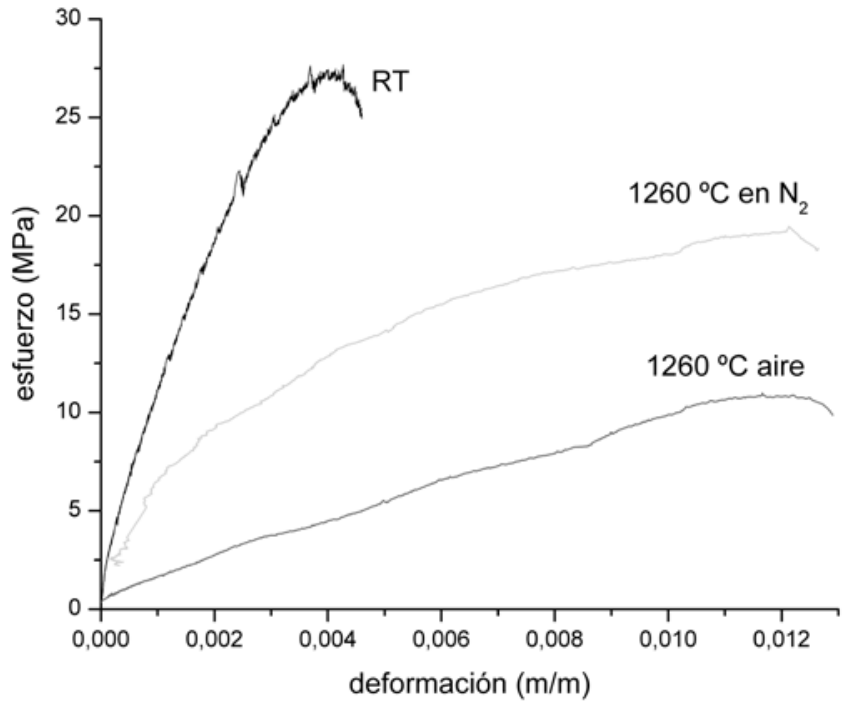

Figura 7. Curvas esfuerzo-deformación de AMC a temperatura ambiente y a $1260{ }^{\circ} \mathrm{C}$ (aire y corriente de $\mathrm{N}_{2}$ ).

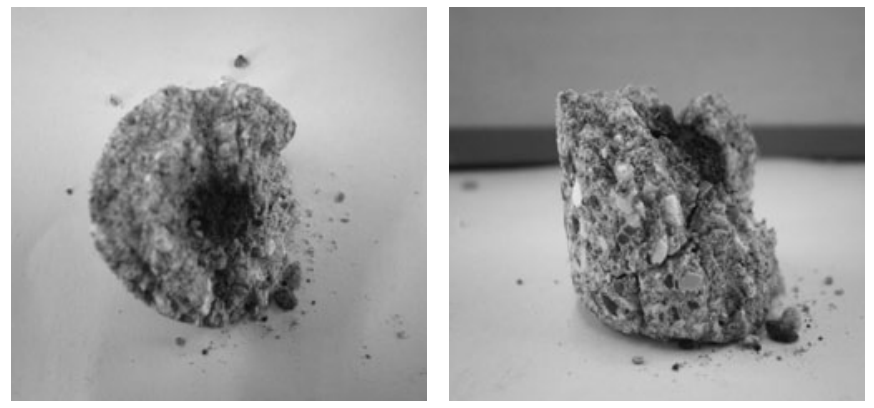

aire

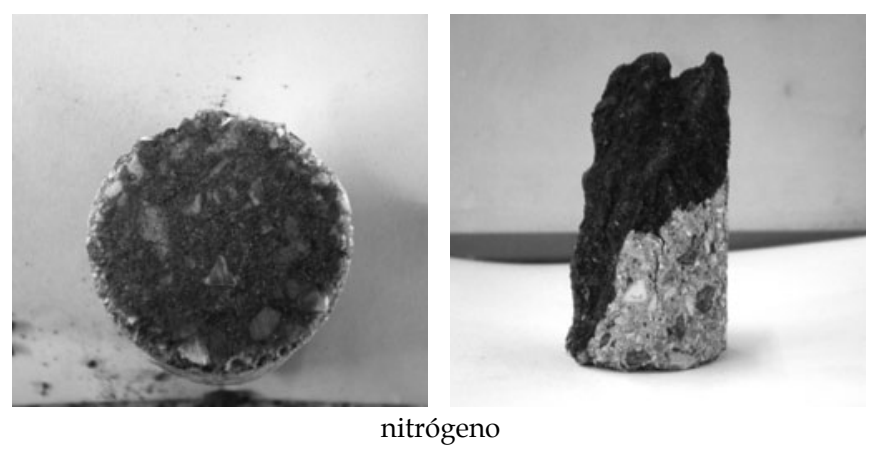

Figura 8. Probetas fracturadas en ensayos mecánicos a $1260^{\circ} \mathrm{C}$. 
(originada en las impurezas de los finos y el grafito) y en los agregados de magnesia sinterizada. En este rango de temperaturas, esta fase se transforma en un fluido de alta viscosidad que favorece la deformación permanente por deslizamiento de partículas y por su propia deformación plástica.

A partir de las curvas esfuerzo-deformación, se determinaron la resistencia $\left(\sigma_{\mathrm{R}}\right)$ y la deformación a la rotura $\left(\varepsilon_{\mathrm{R}}\right)$, tomando como criterio de fractura el máximo valor del esfuerzo registrado y el módulo de Young como la pendiente de la porción lineal de la curva. Los valores de los parámetros se reportan en la Tabla 1. A temperatura ambiente, la resistencia y deformación a la fractura y el módulo elástico del refractario AMC resultan del orden de los encontrados en la literatura para materiales de composiciones similares $[10,12,33,34]$. A $1260{ }^{\circ} \mathrm{C}$ en aire, la disminución de $\sigma_{R} y$ E y el aumento de la deformación de rotura se relacionan principalmente con los cambios que ocurren en atmósfera oxidante en los componentes carbonosos de la fase ligante (resina y grafito), con el aumento significativo de la porosidad aparente. La resina original, de elevada rigidez, se transforma en una estructura carbonosa ('glassy-carbon') con cierto grado de organización [31], lo cual puede contribuir a la disminución del módulo de Young. Respecto a los valores a RT, en corriente de $\mathrm{N}_{2}$ se observa la misma tendencia en la variación de los parámetros que se obtiene en aire, aunque la modificación de los valores es de menor proporción. Este comportamiento puede atribuirse a los mismos factores que determinan la respuesta en aire; sin embargo, el menor desarrollo de los procesos que llevan a la pérdida de componentes hace que el efecto de estos factores sea menor.

El aumento de la porosidad como factor determinante de las propiedades mecánicas a $1260{ }^{\circ} \mathrm{C}$ en aire y en nitrógeno, se debe a la ausencia de fases como $\mathrm{Al}_{4} \mathrm{C}_{3}$ ó AlNC, gracias a las cuales aumenta la resistencia mecánica y la rigidez de este tipo de materiales, tal como ha sido reportado en refractarios similares en el rango de alta temperatura [10, 29]. En ambas atmósferas, aire y nitrógeno, la formación de espinela (identificada por DRX) no tiene efecto positivo sobre las propiedades mecánicas. La mayor cohesión que podría generar este proceso no es suficiente para superar el efecto negativo del incremento en poros y fisuras (en el cual también contribuiría la formación de espinela). Por otra parte, se ha atribuido el empobrecimiento de la respuesta mecánica

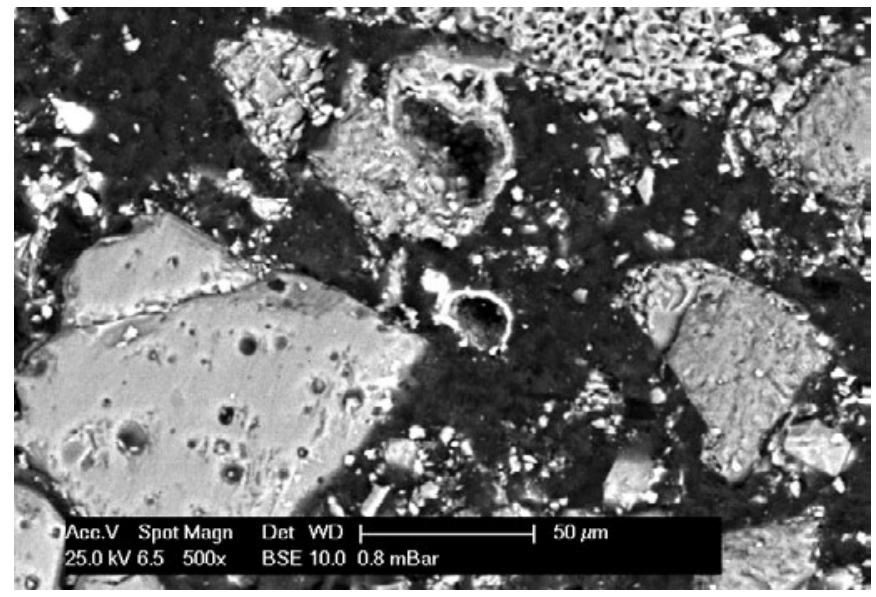

Figura 9. Espinela detectada luego del ensayo a $1260^{\circ} \mathrm{C}$ en aire. a efectos de la morfología tipo espuma que suele presentar la espinela que se forma en este tipo de materiales [10], tal como se observa en la Fig. 9, donde se observa la presencia de espinela (confirmada por EDS) luego del ensayo a $1260^{\circ} \mathrm{C}$ en aire.

\section{CONCLUSIONES}

Se describe una metodología diseñada e implementada para la evaluación mecánica de materiales refractarios a alta temperatura y en condiciones de atmósfera controlada, basada en la medida directa de la variación dimensional de la probeta. La metodología permite determinar curvas esfuerzo-deformación mediante la aplicación de carga en compresión. Se analizan los principales componentes del sistema, extensómetros (capacitivo o de tijeras, según el rango de variación dimensional) y sistemas de aplicación de la carga y de control de atmósfera (desplazamiento por corriente de $\mathrm{N}_{2}$ ).

Se presentan resultados preliminares de la evaluación de materiales refractarios de $\mathrm{Al}_{2} \mathrm{O}_{3}-\mathrm{MgO}-\mathrm{C}$ comerciales de uso en equipamientos de la industria siderúrgica obtenidos empleando la metodología descripta, en diferentes condiciones de temperatura y atmósfera. En condiciones próximas a las de servicio (alta temperatura y carga compresiva), el mejor desempeño del refractario se registró en atmósfera no-oxidante; tanto en aire como en nitrógeno a $1260^{\circ} \mathrm{C}$, se observó una degradación de las propiedades mecánicas del material respecto las obtenidas a temperatura ambiente.

\section{AGRADECIMIENTOS}

Al Dr. Pablo G. Galliano por la provisión de los materiales y al Lic. Leonardo Musante por la orientación en el análisis microestructural por SEM.

\section{REFERENCIAS}

1. C. Alvarez, E.Criado, C. Baudín, "Refractarios de magnesia-grafito", Bol. Soc.Esp.Cerám.Vidr. 31, 5, 397-405. C.F. (1992).

2. E.M.M. Ewais, “Carbon based refractories", Jour.Ceram.Soc.Japan, 112 [10] 517-532 (2004).

3. Cooper, "Refractory application of carbon", Brit.Cer.Trans.Jour., 84, 48-53, (1985).

4. C. Alvarez, E. Criado, C. Baudín, H. Kelichaus, G.S. Duphia: “Hot modulus of rupture automatic testing machine", Proceedings UNITECR'93, São Paulo-Brazil, 435-441. (1993).

5. C. A, Schacht, “Needed fundamental thermomechanical material properties for thermomechanical finite element analysis of refractory structures". In Fundamentals of Refractory Technology, Ceramic Transactions. Vol 125, ed. J. Bennett \& J.D. Smith. The American Ceramic Society, Westerville, 93-101, (2001)

6. A.M. Fitchett and B. Wilshire, "Mechanical properties of carbon-bearing magnesia-I. Resin-bonded magnesia and magnesia-graphite", Br. Ceram. Trans. J., 83, 54-58 (1984).

7. A.M. Fitchett and B. Wilshire, "Mechanical properties of carbon-bearing magnesia-II. Resin-bonded magnesia and magnesia-graphite", Br. Ceram. Trans. J., 83, 59-62 (1984).

8. A.M. Fitchett and B. Wilshire, "Mechanical properties of carbon-bearing magnesia-III. Resin-bonded magnesia and magnesia-graphite", Br. Ceram. Trans. J., 83, 73-76 (1984).

9. D.A. Bell, F.T. Palin: "Measurement of high temperature mechanical properties of refractories containing carbon", Proceedings UNITECR'89, 1219-1124, Anaheim-CA, (1989).

10. C.Baudín, C.Alvarez, R.Moore "Influence of chemical reactions in MgOgraphite refractories: I, Effect on texture and high-temperature mechanical properties". J. Am. Ceram. Soc. 1999; 82(12):3529-38. 
11. C. Baudín, C. Alvarez, R.E. Moore, "Influence of Chemical Reactions in Magnesia-Graphite Refractories, Part II Effects of Graphite and Aluminum Contents in Generic Products", J. Am. Ceram. Soc., 82 (12) 3539-48 (1999).

12. J.M. Robin, Y. Berthaud, N. Schmitt, J. Poirier and D. Themines, "Thermomechanical behaviour of magnesia-carbon refractactories", Brit. Ceram. Trans, 97, 1-10 (1998).

13. P.Williams, \& A. Hagni, "Mineralogical studies of alumina magnesia carbon steel ladle refractories". In Proceeding UNITECR'97 New Orleans, Luisiana USA,183-192,(1997).

14. M. Kamiide, S. Yamamoto, K. Yamamoto, K. Nakahara, \& N. Kido, "Damage of $\mathrm{Al}_{2} \mathrm{O}_{3}-\mathrm{MgO}-\mathrm{C}$ brick for ladle furnace", Jour. Tech. Assoc. Refract. Japan, 21, 252-257, (2001).

15. S. Miglani \& J. J Uchno, "Resin bonded alumina-magnesia-carbon brick for ladles". Proceeding UNITECR'97, New Orleans, Luisiana USA, 193-201, (1997).

16. A. D. Gupta \& K. Vickram, “Development of resin-bonded aluminamagnesia-carbon bricks for steel ladle applications", Interceram ,Vol.48, 5, 307-310, (1999).

17. R.K. Koley, A.V. Rao, S. Askar and S.K. Srivastava, "Development and application of $\mathrm{Al}_{2} \mathrm{O}_{3}-\mathrm{MgO}-\mathrm{C}$ refractory for secondary refining ladle", Proceeding UNITECR'01, (2001).

18. A.A. Nourbakhsh, Sh. Salarian, S.M. Hejazi, S. Shojaiei, F. Golestani-Fard, "Increasing durability of ladle lining refractories by utilizing $\mathrm{Al}_{2} \mathrm{O}_{3}-\mathrm{MgO}-\mathrm{C}$ bricks", Proceeding UNITECR'03, 499-502, (2003).

19. M. A. Camerucci, A.G. Tomba M., A.L. Cavalieri: "Laboratorio de Materiales Cerámicos Estructurales. División Cerámicos-INTEMA. Fac. Ingeniería-UNMdP/CONICET", Cerámica y Cristal, 138, 42-45, (2006).

20. G. A. Rohr, Tesis de grado: "Evaluación mecánica de materiales refractarios con medidas de deformación a alta temperatura y en atmósfera controlada". Ing. en Materiales. Fac. Ingeniería-UNMdP (2006).

21. V. Muñoz, Tesis Doctoral: "Termoquímica y termomecánica de materiales refractarios $\mathrm{Al}_{2} \mathrm{O}_{3}-\mathrm{MgO}-\mathrm{C}$ de uso siderúrgico". Doctorado en Materiales. Fac. Ingeniería-UNMdP (en ejecución desde julio 2008).
22. J.G. Hemrick, "Creep measurement and analysis of refractories". In Fundamentals of Refractory Technology, Ceramic Transactions, ed. J. Bennett \& J.D. Smith. The American Ceramic Society, Westerville, Vol 125,171-193, (2001)

23. A. de S. Jayatilaka, "Fracture of Engineering Brittle Materials". Applied Science Publishers Ltd., London (1979).

24. ASTM E83-94: "Standard Practice for Verification and Classification of Extensometers".

25. Manual Instron: "Especificaciones del extensómetro capacitivo": 3118$230 / 1600{ }^{\circ} \mathrm{C}$.

26. M. ASTM C 133-94: "Standard Test Methods for Cold Crushing Strength and Modulus Rupture of Refractories".

27. T. C. Chou, T. G. Nieh, "Pest desintegration of thin MoSi2 films by oxidation at $500{ }^{\circ} \mathrm{C}^{\prime \prime}$. J. Mater. Sci. 29, 2963-2967, (1994).

28. Manual de Calidad LANAIS 001. Laboratorio de Materiales Estructurales - División Cerámicos -INTEMA.

29. C. Taffin \& J. Poirier, "The behaviour of metal additives in MgO-C and $\mathrm{Al}_{2} \mathrm{O}-\mathrm{C}$ refractories". Interceram, 43, 354-358 [5]/458-460 [6], (1994).

30. M. Rigaud, P. Bombard, X. Li, B. Guéroult, "Phase evolution in various carbon-bonded nasic refrctories", Proceedings of the UNITECR'93, 360371.

31. DIN EN 993-1(DIN 51056). Method of test for dense shaped refractory products. Determination of bulk density, apparent porosity and true porosity (DIN 51056) 1995.

32. B.Rand, \& B. McEnaney, "Carbon binders from polymeric resins and pitch Part I-Pyrolisis behaviour and structure of the carbons", Br. Ceram.Trans. J. 84, 175-165, (1985).

33. N. Schmitt, Y. Berthaud \& J.Poirier, "Tensile behavior of magnesia carbon refractories". Jour. Eur. Ceram. Soc., 20, 2239-2248. (2000)

34. L. Musante, V. Muñoz, M.H. Labadie, A.G. Tomba Martinez "High temperature mechanical behavior of $\mathrm{Al}_{2} \mathrm{O}_{3}-\mathrm{MgO}-\mathrm{C}$ refractories for steelmaking use", Cer. Int., in prensa. 